\title{
Study of the object of the natural-technical system and determination of the static characteristics of ventilation
}

\author{
Igor I. Bosikov*, Roman V. Klyuev, Boris V. Dzeranov, and Marat V. Tekiev \\ North Caucasian Institute of Mining and Metallurgy (State Technological University), Industrial \\ Power Supply Department, 44 str. Nikolaeva, Russia
}

\begin{abstract}
The article describes the analysis of the operation of existing production facilities for the extraction and processing of deposits. The study of the effective functioning of the natural-technical system in the extraction of a useful component of deposits is an extremely urgent and promising task. The purpose of the research is to study the object of the natural-technical system and determine the type of static ventilation characteristics. The tasks will be solved on the basis of a comprehensive study of the object of the natural-technical system. Experimental data necessary for constructing static characteristics associated with conducting a special experiment on the production site that violates the normal mode of ventilation is obtained. The research and development of a method for determining the static characteristics of ventilation objects, according to normal operation, which are obtained without interrupting the technological process of extracting the useful component.
\end{abstract}

\section{Scientific significance}

At the present stage of the development of the raw material base of the Russian Federation, a considerable acceleration of the rates of technical progress and an increase in the efficiency of the production of mining enterprises are planned. The fulfillment of this task is possible on condition of technical re-equipment based on new high-performance equipment, progressive technological schemes, complex mechanization and automation of all processes, further improvement of working conditions and safety engineering.

A large number of works in this field is of a qualitative nature only, describing the results of experiments with a sharp adjustment of the air flow in the area. Particular attention is paid to the phenomenon of a short-term change in the concentration of methane in synchronism with the air flow, which was called the "spike" of methane concentration. In a number of works it was noted that transient gas-dynamic processes can proceed without a "spike" of methane concentration.

\footnotetext{
* Corresponding author: igor.boss.777@mail.ru
} 
This group of studies includes the works of I.A. Ryzhenko, I.P. Ignatenko, M.A. Patrushev, A.S. Tsyrulnikov, E.A. Litvinenko, B.E. Gretsangera, O.I. Kasimov, L.A. Puchkov and others.

In the works of F.S. Klebanov, I.A. Ryzhenko, a theoretical generalization is given by an analytical study of transient gas-dynamic processes with "spikes" of methane concentration. The main drawback of the obtained mathematical dependences is that they do not take into account changes in transient gas-dynamic processes in time.

\section{Formulation of the problem}

The following main tasks are set in the present paper: to develop methods for obtaining a mathematical description of the ventilation of mining sites in static and dynamic; taking into account the specific features of the random air-gasdynamic processes, to develop methods for controlling the parameters of the mine atmosphere, the primary processing of information on the generation of control actions by the gas situation of the mining site; taking into account the complex network nature of the ventilation system, to develop simple methods for its encoding, efficient methods, as well as algorithms for solving basic topological problems; to develop methods and algorithms for solving the problems of natural flow distribution and optimal control of air distribution in ventilation networks of mines, effective in speed, universality and economy.

\section{Theoretical part}

Influence of production processes of a mining site on the statistical characteristics of airgasdynamic processes.

In the mathematical description of the dynamics of the ventilation system of the mining site of the mine as a control object by the gas factor, methods of statistical dynamics are widely used [8-10]. The initial experimental material can be the implementation of the air flow processes $Q(t)$ and the methane concentration $C(t)$, written as deviations from their mathematical expectations:

$$
\begin{gathered}
C^{0}(t)=C(t)-C \\
Q^{0}(t)=Q(t)-Q
\end{gathered}
$$

where $C, Q$ - mathematical expectations of processes $C(t)$ and $Q(t)$.

It is established in the paper that the realizations of the processes $C^{0}(t)$ and $Q^{0}(t)$ are stationary and ergodic in observation intervals of 3-5 days.

On long observation intervals (weeks, months), the gas dynamic processes acquire nonstationary character, i.e. their statistical characteristics (mathematical expectations, correlation functions) vary with time. This is confirmed by the experimental results of the works and is explained by the influence of changes in the mining and geological and mining conditions of the development of coal seams.

The conducted researches established that the production processes of the mining areas cause fluctuations in the air-gas dynamic processes. Changes in gas evolution mainly depend on the mode of operation of the longwall (alternating production and repair shifts) and the technological cycle for the extraction of the useful component.

Production processes in the mining area cause not only fluctuations in gas evolution, but also changes in the air flow over a wide range. This is explained by the fact that the existing technology of operations, involving the sequential execution of all operations of the 
purification cycle, significantly changes the aerodynamic resistance of the mining site during the technological cycle (by 30-40\%) [2].

On the basis of the foregoing, the processes in the deviations of $C^{0}(t)$ and $Q^{0}(t)$ can be represented as:

$$
\begin{array}{r}
C^{0}(t)=C_{c}^{0}(t)+C_{k}^{0}(t) \\
Q^{0}(t)=Q_{c}^{0}(t)+Q_{k}^{0}(t),
\end{array}
$$

where $C_{c}^{0}(t), Q_{c}^{0}(t)$ are purely random processes of methane concentration and air flow; $C_{k}^{0}(t), Q_{k}^{0}(t)$ are processes of methane concentration and air flow, the fluctuations of which are caused by the production processes of the site.

To verify the validity of relations (3) and (4), we use the methods of random functions [12], based on the calculation of correlation functions. Such an approach is rational because it allows not only to solve the task, but also to use the obtained statistical characteristics of the processes $C^{0}(t)$ and $Q^{0}(t)$ to determine the dynamic characteristics of the objects of airing.

The principle possibility of applying correlation functions for the analysis of the processes $C^{0}(t)$ and $Q^{0}(t)$ is explained by the fact that they allow us to extract the periodic components of the processes under study in a fairly large time interval.

To calculate the correlation and correlation functions, we use the formulas [1-3]:

$$
\begin{aligned}
& R_{C}(\tau)=\frac{1}{T} \int_{0}^{T} C^{0}(t) \cdot C^{0}(t+\tau) d t \\
& R_{Q}(\tau)=\frac{1}{T} \int_{0}^{T} Q^{0}(t) \cdot Q^{0}(t+\tau) d t \\
& R_{C Q}(\tau)=\frac{1}{T} \int_{0}^{T} C^{0}(t) \cdot Q^{0}(t+\tau) d t
\end{aligned}
$$

where $R_{C}(\tau), R_{Q}(\tau)$ - are correlation functions of processes $C^{0}(t)$ and $Q^{0}(t) ; R_{C Q}(\tau)$ - is correlation function of processes $C^{0}(t)$ and $Q^{0}(t) ; \tau$ - is the interval of the shift of realizations, min; $T, t$ - interval of observation, min.

Functions $R_{C}(\tau), R_{Q}(\tau)$ and $R_{C Q}(\tau)$ were calculated using the developed standard program. The values of the obtained functions $R_{C}(\tau), R_{Q}(\tau)$ and $R_{C Q}(\tau)$ decrease with increasing distance from zero and for $\tau>5-10$ hours they acquire a periodic character (the so-called "tails" of the correlation functions). The last ones can be oscillations due to random errors in the determination of correlation functions. Random errors include not only errors due to measurement errors, time quantization of the processes $C^{0}(t)$ and $Q^{0}(t)$ and the finite interval of observation, but also random perturbations with periods $T<1$ hour, which are caused, for example, by the work of lifting the shaft, the opening of ventilation doors, etc.

To reduce the value of the random error amplitude, the experimental correlation and intercorrelation functions were smoothed by the moving average method with an interval $T_{c}=1-2$ hours. In this case, the amplitudes of vibrations with periods $T<T_{c}$ are attenuated (decreasing) by a factor of 5-10, and the amplitudes of low-frequency vibrations $\left(T>4 T_{C}\right)$ decrease by only $5 \%$ [3-8]. 


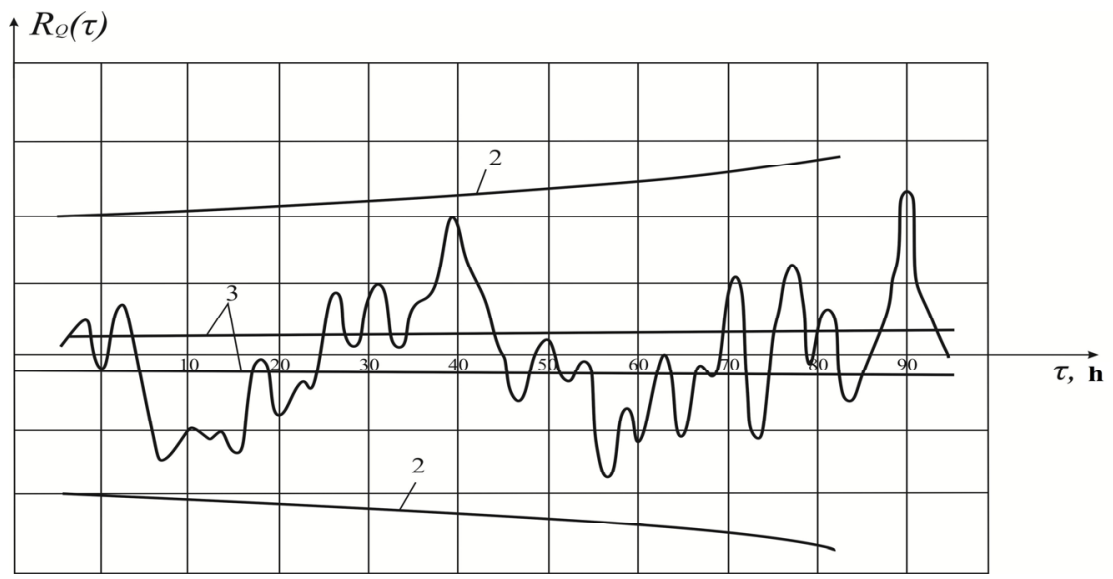

$a$

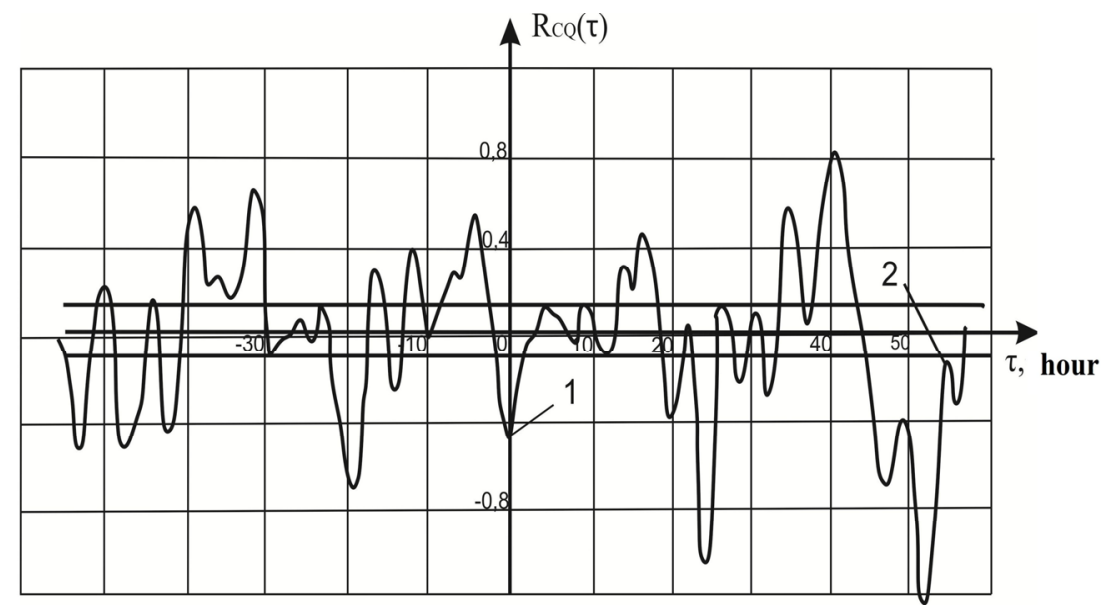

$b$

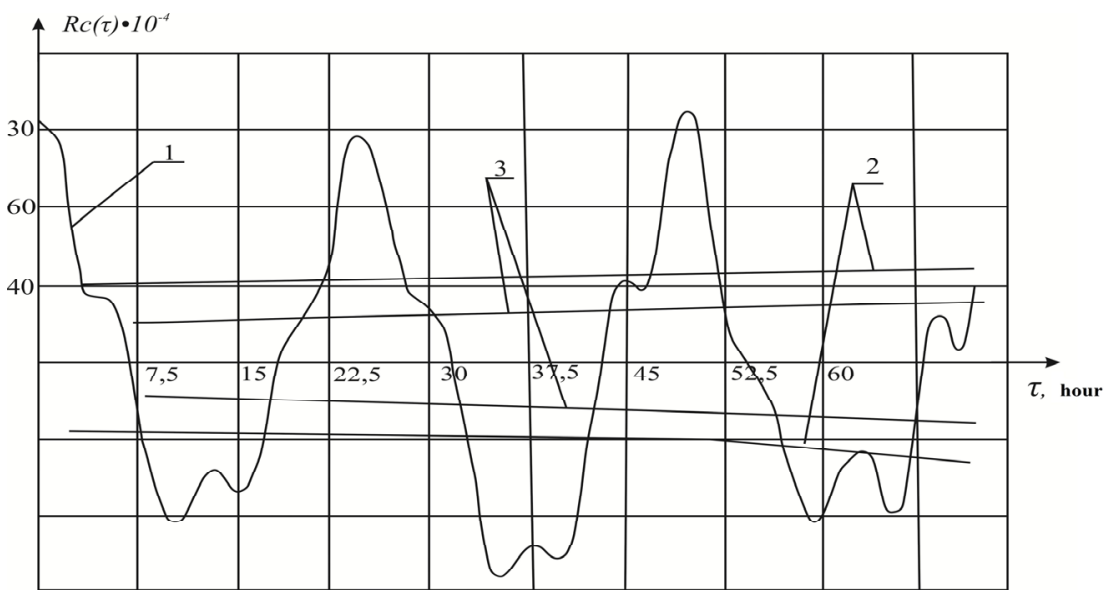

$c$

Fig. 1. Graphs of correlation functions: $a$-correlation function $C(t)$, curve $1 ; b$-intercorrelation function, curve $1 ; c$ - correlation function $Q(t)$, curve $1 ; 2,3$ - levels of significance. 
The graphs of Figure 1 show the experimental correlation functions of methane concentration (Fig. 1a) and the air flow rate (Fig. 1b), the intercorrelation function (Figure 1c) between the methane concentration and air flow, constructed from the results of airgasdynamic processes in site 4 of shaft 58 .

The graphs show that after smoothing, the "tailings" of the correlation functions remain and their amplitudes do not decrease with increasing $\tau$, as it should have been if they were due to errors in the calculation of the correlation functions of purely random processes.

As can be seen from Fig. 2, the correlation functions of purely random processes $R_{C_{c}}(\tau)$ and $R_{Q_{c}}(\tau)$ tend to zero with increasing $\tau$ and, consequently, condition (20) is fulfilled. Thus, purely random stationary processes $C_{c}{ }^{0}(t)$ and $Q_{c}{ }^{0}(t)$ possess have an ergodic property.

The correlation functions of the components due to the effect on the gas evolution of the production processes of the site have vibrational, close to periodic character. Their peculiarity is that they are not damped, this is traced in the interval 24-72 hours, and that there is no strict periodicity, which manifests itself not only in the amplitude change, but also in the composition of the frequencies. These features are quite clearly visible from the graphs of Fig. 1.

The components of $C_{\kappa}{ }^{0}(t)$ and $Q_{\kappa}{ }^{0}(t)$, due to the influence of production processes, can be represented as:

$$
\begin{array}{r}
C_{\kappa}^{0}(t)=\sum_{i=1}^{n} C_{i} \sin \left(\omega_{i} t+\phi_{i}\right), \\
Q_{\kappa}^{0}(t)=\sum_{i=1}^{n} Q_{i} \sin \left(\omega_{i} t+\psi_{i}\right) .
\end{array}
$$

If the processes $C_{\kappa}^{0}(t)$ and $Q_{\kappa}^{0}(t)$, are deterministic, i.e. the values $C_{i}=$ const, $Q_{i}=$ const, $\varphi_{i}=$ const, $\psi_{i}=$ const then their correlation functions have the form:

$$
R_{C_{k}}(\tau)=\frac{1}{2} \sum_{i=1}^{n} C_{i}^{2} \cos \omega_{i} \tau
$$

In this case, the correlation functions ( 8 and 9$)$ must be strictly periodic, which does not correspond to the graphs of the experimental correlation functions $R_{C}(\tau)$ and $R_{Q}(\tau)$ in Fig.2.

$$
R_{Q_{k}}(\tau)=\frac{1}{2} \sum_{i=1}^{n} Q_{i}^{2} \cos \omega_{i} \tau
$$

If the amplitudes $C_{i}$ and $Q_{i}$ in expressions $(10,11)$ are random processes, and their correlation functions in the simplest case are described by expressions $\frac{1}{2} C_{i}{ }^{2} e^{-\alpha \tau}$ and $\frac{1}{2} Q_{i}^{2} e^{-\alpha \tau}$ then the correlation functions of processes $C_{\kappa}{ }^{0}(t)$ and $Q_{\kappa}{ }^{0}(t)$ :

$$
\begin{aligned}
& R_{C_{\kappa}}(\tau)=\frac{1}{2} \sum_{i=1}^{n} C_{i}^{2} e^{-\alpha \tau} \cos \omega_{i} \tau, \\
& R_{Q_{\kappa}}(\tau)=\frac{1}{2} \sum_{i=1}^{n} Q_{i}^{2} e^{-\alpha \tau} \cos \omega_{i} \tau .
\end{aligned}
$$




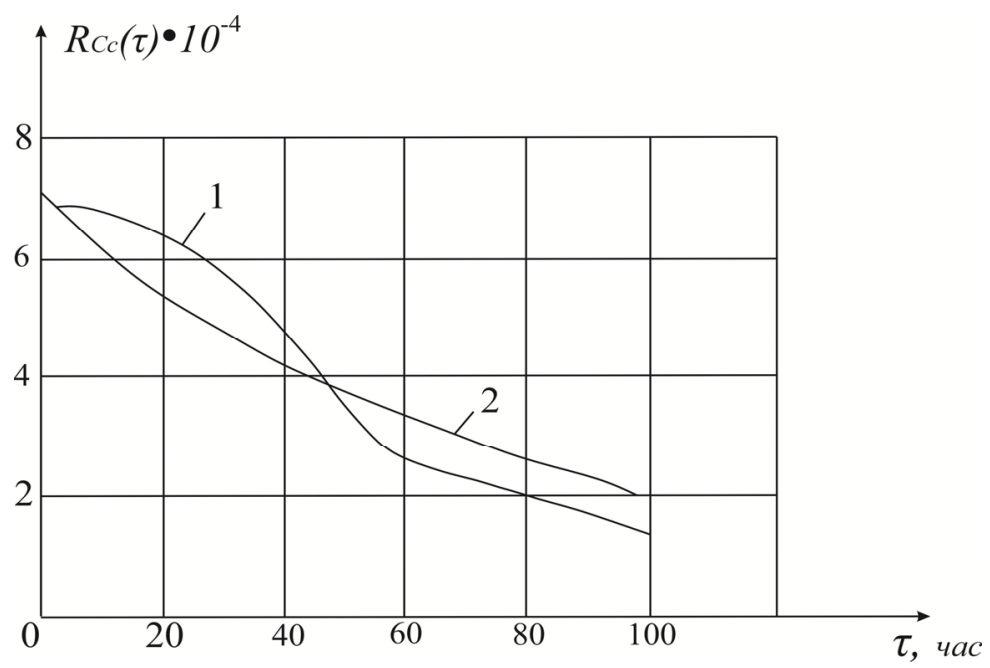

Fig. 2. Graph of the correlation function of the random component of the process $C_{c}(t)$, curve $1 ; 2-$ approximating curve.

The correlation functions (11) and (12) are damped functions, which also does not correspond to the experimental curves of the function $R_{C}(\tau)$ and $R_{Q}(\tau)$.

If the amplitudes $C_{i}$ and $Q_{i}$ are random variables and the stationarity conditions of the processes $C_{\kappa}{ }^{0}(t)$ and $Q_{\kappa}{ }^{0}(t)$ :

$$
M\left\{C_{i}\right\}=M\left\{Q_{i}\right\}=0, M\left\{C_{i}^{2}\right\}=\frac{1}{2} D_{C_{i}}, M\left\{Q_{i}^{2}\right\}=\frac{1}{2} D_{Q_{i}},
$$

where $M$ is the symbol of mathematical expectation, the correlation functions of processes (7) and (8) will have the form:

$$
\begin{aligned}
& R_{C_{\kappa}}(\tau)=\frac{1}{2} \sum_{i=1}^{n} D_{C_{i}} \cos \omega_{i} \tau, \\
& R_{Q_{\kappa}}(\tau)=\frac{1}{2} \sum_{i=1}^{n} D_{Q_{i}} \cos \omega_{i} \tau .
\end{aligned}
$$

The correlation functions (15) and (16) have an undamped character, which differs from the strict periodicity.

The mutual correlation function $R_{C_{k} Q_{k}}(\tau)$ in this case has the form:

$$
R_{C_{k} Q_{k}}(\tau)=\frac{1}{2} \sum_{i=1}^{n} C_{i} \cdot Q\left[\cos \left(\varphi_{i}-\psi_{i}\right) \cdot \cos \omega_{i} \tau \pm \sin \left(\varphi_{i}-\psi_{i}\right) \cdot \sin \omega_{i} \tau\right]
$$

\section{Results of experimental studies}

It can be seen from formula (17) that the value of the intercorrelation function $R_{C_{k} Q_{k}}(\tau)$ depends on the random phase $\left(\varphi_{i}-\psi_{i}\right)$ shift between the processes $C_{k}^{0}(t)$ and 
$Q_{\kappa}{ }^{0}(t)$. This circumstance makes it possible to explain the distortions in the form of the experimental intercorrelation function of purely random processes $R_{C_{k} Q_{k}}(\tau)$.

Thus, the correlation functions $R_{C_{k}}(\tau), R_{Q_{k}}(\tau)$ and $R_{C_{k} Q_{k}}(\tau)$ completed with the help of mathematical models (7 and 8), when their amplitudes $C_{i}, Q_{i}$ and phases $\varphi_{i}$ and $\psi_{i}$ are random variables, best reflect the character of the change in the experimental correlation functions of the components $C_{\kappa}{ }^{0}(t)$ and $Q_{\kappa}{ }^{0}(t)$ due to the influence of the production processes of the site.

Since the parameters of the processes $C_{\kappa}{ }^{0}(t)$ and $Q_{\kappa}{ }^{0}(t)$ vary randomly, these processes should be referred to quasideterministic random processes [7-10].

Analysis of the statistical properties of the constituent processes of $C_{\kappa}{ }^{0}(t)$ and $Q_{\kappa}{ }^{0}(t)$ has shown that purely random processes the concentration of methane $C_{\kappa}{ }^{0}(t)$ and air flow $Q_{\kappa}{ }^{0}(t)$ are stationary and ergodic processes with a normal distribution law in intervals of 3-5 days, and quasideterministic random processes $C_{\kappa}{ }^{0}(t)$ and $Q_{\kappa}{ }^{0}(t)$ are stationary processes with a normal distribution law.

\section{Conclusion}

Experimental data which are necessary for constructing static characteristics associated with conducting a special experiment on the production site that violates the normal mode of airing are obtained. The research and development of a method for determining the static characteristics of airing objects, according to normal operation, which are obtained without interrupting the technological process of extracting the useful component were carried out.

\section{References}

1. N. N. Moiseev, Mathematical problems of system analysis (Nauka, Moscow, 1981)

2. A. S. Rykov, Models and methods of system analysis: decision making and optimization (MISIS, Moscow, 2005)

3. L. A. Puchkov, Aerodynamics of underground exhausted spaces (Publishing house MGGU, Moscow 1993)

4. D. Sbárbaro, René del Villar, Advanced Control and Supervision of Mineral Processing Plants (Springer, London, 2010)

5. A. Remes, Advanced Process Monitoring and Control Methods in Mineral Processing Applications (Aalto University, Espoo, 2012)

6. P. G. Belov, System analysis and modeling of hazardous processes in the technosphere (Publishing Center "Academy", Moskow, 2003)

7. R.V. Klyuev, I. I. Bosikov, V. B.Kelekhsaev, International Conference on Industrial Engineering, Applications and Manufacturing (ICIEAM), DOI: 10.1109/ICIEAM.2017.8076113, 1-5 (2017)

8. I. I. Bosikov, R.V. Klyuev, V. B.Kelekhsaev, International Conference on Industrial Engineering, Applications and Manufacturing (ICIEAM), DOI: 10.1109/ICIEAM.2017.8076114, 1-6, (2017)

9. R. B. Youn, R. V. Klyuev, I. I. Bosikov, B. V. Dzeranov, Sustainable Development of Mountain Territories, 9 2(32) 172-178 (2017)

10. N. Sh. Kremer, Probability theory and mathematical statistics (UNITEDONE, Moskow, 2004) 